\title{
Thermally Activated Delayed Fluorescence in Fullerenes
}

\author{
Garlos Baleizão and Mário N. Berberan-Santos \\ Centro de Química-Física Molecular, Instituto Superior Técnico, Lisbon, Portugal
}

\begin{abstract}
This report reviews the thermally activated delayed fluorescence (TADF) displayed by fullerenes. From the analysis of the steady-state data, time-resolved data, or by a combination of both, it is possible to determine several important photophysical parameters of fullerenes. Herein we also cover the development of temperature and oxygen sensors based on the TADF effect exhibited by fullerene $\mathrm{C}_{70}$. Despite the work already carried out, knowledge of the photophysics of fullerenes and derivatives is still incomplete, and much remains to be done in this area and in the improvement of sensor systems based on fullerenes.
\end{abstract}

Key words: fullerenes; $\mathrm{C}_{60} ; \mathrm{C}_{70}$; thermally activated delayed fluorescence; temperature sensing; oxygen sensing

\section{Introduction}

The discovery of fullerenes in $1985^{1}$ and their production in macroscopic amounts after $1990^{2}$ opened a new field of research. Applications of fullerenes in areas, such as energy, materials, biopharmaceuticals, optics, and electronics, have started to appear in recent years. The most common fullerenes are $\mathrm{C}_{60}$ and $\mathrm{C}_{70}$, three-dimensional carbon structures that can be viewed either as large carbon molecules or as tiny nanoparticles with welldefined composition and shape. Their photophysical and photochemical properties result from the many delocalized $\pi$ electrons present and from the high symmetry and curvature of the structures. The photophysics of fullerenes has been the subject of considerable investigation. ${ }^{3-5}$ One of the most characteristic and (to us) interesting photophysical properties of $\mathrm{C}_{60}, \mathrm{C}_{70}$, and derivatives, first observed in our group, is a second mechanism for fluorescence, which is called thermally activated delayed fluorescence (TADF). This phenomenon, which occurs in a few fluorescent molecules, is usually weak; however, for fullerenes it is strong, especially for $\mathrm{C}_{70}$. Herein, we briefly review the TADF of fullerenes and the development of temperature and oxygen sensors based on this property.

Address for correspondence: Mário N. Berberan-Santos, Centro de Química-Física Molecular, Instituto Superior Técnico, Av. Rovisco Pais, 1049-001 Lisbon, Portugal. Voice: +351-218419254; fax: +351218464455.

berberan@ist.utl.pt

\section{TADF: Fundamental Aspects}

Two distinct unimolecular mechanisms exist for molecular fluorescence: prompt fluorescence $(\mathrm{PF})$ and TADF $^{6,7}$ In the PF mechanism, emission occurs after $\mathrm{S}_{\mathrm{n}} \leftarrow \mathrm{S}_{0}$ absorption and excited state relaxation to $\mathrm{S}_{1}$. The TADF mechanism takes place by way of the triplet manifold: After excitation and once $\mathrm{S}_{1}$ is attained, intersystem crossing (ISC) to the triplet manifold ( $\mathrm{T}_{1}$ or a higher triplet) occurs, followed by a second ISC from $T_{1}$ back to $S_{1}$, and by fluorescence emission proper. The cycle $\mathrm{S}_{1} \rightarrow \mathrm{T}_{1} \rightarrow \mathrm{S}_{1}$ may be repeated several times before fluorescence finally takes place. TADF is significant only when the quantum yield of triplet formation $\left(\Phi_{\mathrm{T}}\right)$ and the quantum yield of singlet formation $\left(\Phi_{\mathrm{S}}\right)$ are both high. ${ }^{8}$ This outcome in turn implies a small energy gap between $\mathrm{S}_{1}$ and $\mathrm{T}_{1}$ $\left(\Delta E_{\mathrm{ST}}\right)$, a long $\mathrm{T}_{1}$ lifetime, and not too low a temperature. ${ }^{8}$ For a given fluorophore, TADF is usually much weaker than its PF. Although known for many years, TADF continues to be a rare phenomenon, with a few observations in some xanthene dyes ${ }^{6,9,10}$ aromatic ketones ${ }^{11,12}$ and thiones,${ }^{13,14}$ metal porphyrins, ${ }^{15}$ and aromatic hydrocarbons. ${ }^{16-18}$

The remarkable photophysical properties of fullerene $\mathrm{C}_{70}$, specifically for the $\Phi_{\mathrm{T}}$ very close to $1,{ }^{19}$ the small $\Delta E_{\mathrm{ST}}$ gap, ${ }^{20}$ and the long intrinsic phosphorescence lifetime, ${ }^{21}$ led to the discovery of an exceptionally strong TADF in this molecule. ${ }^{8} \mathrm{C}_{60}{ }^{22}$ and some $\mathrm{C}_{60}$ derivatives, ${ }^{23,24}$ as well as one $\mathrm{C}_{70}$ derivative, ${ }^{25}$ also exhibit TADF, but weaker than that of $\mathrm{C}_{70}$.

The simplest model for TADF in the condensed phases is a three-state system that can be represented by 


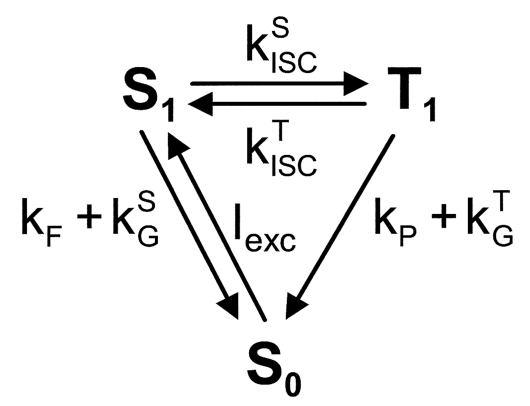

SCHEME 1. Kinetic scheme for TADF.

SCHEME 1, where $I_{\text {exc }}$ is the excitation intensity; $k_{\mathrm{F}}$ and $k_{\mathrm{P}}$ are the radiative rate constants for fluorescence and phosphorescence, respectively; $k_{\mathrm{G}}^{\mathrm{S}}$ and $k_{\mathrm{G}}^{\mathrm{T}}$ are the nonradiative rate constants for deactivation to the ground state (internal conversion from $\mathrm{S}_{1}$ and ISC from $\mathrm{T}_{1}$, respectively); and $k_{\mathrm{ISC}}^{\mathrm{S}}$ and $k_{\mathrm{ISC}}^{\mathrm{T}}$ are the ISC rate constants for singlet-to-triplet and triplet-to-singlet conversion, respectively. Owing to the relative energies of $S_{1}$ and $\mathrm{T}_{1}$, the triplet-to-singlet ISC rate constant always corresponds to an activated process that is strongly temperature dependent ${ }^{6,8,26,27}$ :

$$
k_{\mathrm{ISC}}^{\mathrm{T}}=A \exp \left(-\frac{\Delta E_{\mathrm{ST}}}{R T}\right)
$$

For strong TADF to occur, the following inequalities need to be met: $k_{\mathrm{ISC}}^{\mathrm{S}} \gg k_{\mathrm{F}}+k_{\mathrm{G}}^{\mathrm{S}}$ and $k_{\mathrm{ISC}}^{\mathrm{T}} \gg k_{\mathrm{P}}+k_{\mathrm{G}}^{\mathrm{T}}$. Usually it is also observed that $k_{\mathrm{ISC}}^{\mathrm{S}} \gg k_{\mathrm{ISC}}^{\mathrm{T}}$ and $k_{\mathrm{G}}^{\mathrm{T}}$ $\gg k_{\mathrm{P}}$.

The time evolution of the $S_{1}$ and $T_{1}$ populations is given by the following coupled equations, ${ }^{28}$ where for simplicity the square brackets representing the concentrations are omitted:

$$
\begin{aligned}
S_{1}(t) & =I_{\text {exc }}(t) \otimes \exp \left(-t / \tau_{\mathrm{F}}\right) \\
& +k_{\mathrm{ISC}}^{\mathrm{T}} T_{1}(t) \otimes \exp \left(-t / \tau_{\mathrm{F}}\right) \\
T_{1}(t) & =k_{\mathrm{ISC}}^{\mathrm{S}} S_{1}(t) \otimes \exp \left(-t / \tau_{\mathrm{P}}\right)
\end{aligned}
$$

Here $\otimes$ stands for the convolution between two functions, $\quad f \otimes g=\int_{0}^{t} f(u) g(t-u) d u ; \tau_{\mathrm{F}}=1 /\left(k_{\mathrm{F}}+\right.$ $\left.k_{\mathrm{G}}^{\mathrm{S}}+k_{\mathrm{ISC}}^{\mathrm{S}}\right)$ is the (prompt) fluorescence lifetime; and $\tau_{\mathrm{P}}=1 /\left(k_{\mathrm{P}}+k_{\mathrm{G}}^{\mathrm{T}}+k_{\mathrm{ISC}}^{\mathrm{T}}\right)$ is called here the phosphorescence lifetime. These two lifetimes have direct experimental meaning only in the absence of reversibility; otherwise, fluorescence and phosphorescence no longer have single exponential decays, as will be discussed below. The low-temperature phosphorescence lifetime is $\tau_{\mathrm{P}}^{0}=1 /\left(k_{\mathrm{P}}+k_{\mathrm{G}}^{\mathrm{T}}\right)$. For rigid molecules, the temperature dependence of $k_{\mathrm{G}}^{\mathrm{T}}$ is dictated mainly by external effects, that is, interactions with the solvent and other solutes present, such as oxygen and impurities, and therefore $k_{\mathrm{G}}^{\mathrm{T}}$ is expected to change moderately with temperature in a deoxygenated and photochemically inert solid medium. ${ }^{29}$

SCHEME 1 is isomorphous to the monomer-excimer scheme (without transient effects ${ }^{30}$ ) and therefore has the same general solution. This solution can be obtained by inserting EQUATION (3) into EQUATION (2),

$$
\begin{gathered}
S_{1}(t)=I_{e x c}(t) \otimes \exp \left(-t / \tau_{\mathrm{F}}\right)+k_{\mathrm{ISC}}^{\mathrm{S}} k_{\mathrm{ISC}}^{\mathrm{T}} S_{1}(t) \\
\otimes \exp \left(-t / \tau_{\mathrm{P}}\right) \otimes \exp \left(-t / \tau_{\mathrm{F}}\right)
\end{gathered}
$$

and then by repeated substitution of the left-hand side on the right-hand side, ${ }^{26}$

$$
\begin{aligned}
S_{1}(t)= & I_{\text {exc }}(t) \otimes \exp \left(-t / \tau_{\mathrm{F}}\right) \\
& +k_{\mathrm{ISC}}^{\mathrm{S}} k_{\mathrm{ISC}}^{\mathrm{T}} I_{e x c}(t) \otimes \exp \left(-t / \tau_{\mathrm{F}}\right) \\
& \otimes \exp \left(-t / \tau_{\mathrm{P}}\right) \otimes \exp \left(-t / \tau_{\mathrm{F}}\right)+ \\
& +\left(k_{\mathrm{ISC}}^{\mathrm{S}} k_{\mathrm{ISC}}^{\mathrm{T}}\right)^{2} I_{e x c}(t) \\
& \otimes \exp \left(-t / \tau_{\mathrm{F}}\right) \otimes \exp \left(-t / \tau_{\mathrm{P}}\right) \\
& \otimes \exp \left(-t / \tau_{\mathrm{F}}\right) \otimes \exp \left(-t / \tau_{\mathrm{P}}\right) \\
& \otimes \exp \left(-t / \tau_{\mathrm{F}}\right)+\ldots \ldots
\end{aligned}
$$

hence, the first term for the singlet decay can be associated with $\mathrm{PF}$ (zero $\mathrm{S}_{1} \rightarrow \mathrm{T}_{1} \rightarrow \mathrm{S}_{1}$ cycles), and the remaining terms with delayed fluorescence, the $n$th term resulting from $n-1$ $\mathrm{S}_{1} \rightarrow \mathrm{T}_{1} \rightarrow \mathrm{S}_{1}$ cycles. Analogous results can be obtained for the triplet decay. The singlet decay, EQUATION (5), simplifies into a sum of two exponentials of time, and the triplet decay into a difference of the same two exponentials ${ }^{31}$ :

$$
\begin{aligned}
S_{1}(t)= & \frac{S_{1}(0)}{\lambda_{2}-\lambda_{1}}\left[\left(\lambda_{2}-X\right) \exp \left(-\lambda_{1} t\right)\right. \\
& \left.+\left(X-\lambda_{1}\right) \exp \left(-\lambda_{2} t\right)\right]
\end{aligned}
$$

$$
T_{1}(t)=\frac{k_{\mathrm{ISC}}^{\mathrm{S}} S_{1}(0)}{\lambda_{2}-\lambda_{1}}\left[\exp \left(-\lambda_{1} t\right)-\exp \left(-\lambda_{2} t\right)\right]
$$

where

$$
\lambda_{1,2}=\frac{1}{2}\left\{X+r \mp \sqrt{(r-X)^{2}+4 k_{\mathrm{ISC}}^{\mathrm{S}} k_{\mathrm{ISC}}^{\mathrm{T}}}\right\}
$$

with

$$
X=\frac{1}{\tau_{\mathrm{F}}}
$$

and

$$
r=\frac{1}{\tau_{\mathrm{P}}^{0}}+k_{\mathrm{ISC}}^{\mathrm{T}}
$$

When interconversion between the singlet and triplet emissive states occurs many times before photon emission or nonradiative decay can take place, a fast preequilibrium between $S_{1}$ and $T_{1}$ is established, and 
for sufficiently long times both $\mathrm{S}_{1}$ and $\mathrm{T}_{1}$ decay with a common rate constant given by ${ }^{32}$

$$
k=\frac{k_{\mathrm{ISC}}^{\mathrm{S}}}{k_{\mathrm{ISC}}^{\mathrm{S}}+k_{\mathrm{ISC}}^{\mathrm{T}}} k_{\mathrm{G}}^{\mathrm{T}}+\frac{k_{\mathrm{ISC}}^{\mathrm{T}}}{k_{\mathrm{ISC}}^{\mathrm{S}}+k_{\mathrm{ISC}}^{\mathrm{T}}} k_{\mathrm{G}}^{\mathrm{S}}
$$

Given the inequalities mentioned, EQUATION (11) simplifies to

$$
k=\frac{1}{\tau_{\mathrm{DF}}}=k_{\mathrm{G}}^{\mathrm{T}}+\left(1-\Phi_{\mathrm{T}}\right) k_{\mathrm{ISC}}^{\mathrm{T}}
$$

where $\Phi_{\mathrm{T}}$ is the quantum yield of triplet formation, $\Phi_{\mathrm{T}}=k_{\mathrm{ISC}}^{\mathrm{S}} /\left(k_{\mathrm{F}}+k_{\mathrm{G}}^{\mathrm{S}}+k_{\mathrm{ISC}}^{\mathrm{S}}\right)$, and $\tau_{\mathrm{DF}}$ is the delayed fluorescence (and phosphorescence) lifetime.

The fluorescence quantum yield is given by

$$
\Phi_{\mathrm{F}}=\Phi_{\mathrm{PF}}+\Phi_{\mathrm{DF}}
$$

where the quantum yields for prompt $\Phi_{\mathrm{PF}}$ and delayed $\Phi_{\mathrm{DF}}$ fluorescence obey the following relation ${ }^{8}$ :

$$
\frac{\Phi_{\mathrm{DF}}}{\Phi_{\mathrm{PF}}}=\frac{I_{\mathrm{DF}}}{I_{\mathrm{PF}}}=\frac{1}{\frac{1}{\Phi_{\mathrm{S}} \Phi_{\mathrm{T}}}-1}
$$

and the quantum yield of singlet formation is defined by

$$
\Phi_{\mathrm{S}}=\frac{k_{\mathrm{ISC}}^{\mathrm{T}}}{k_{\mathrm{P}}+k_{\mathrm{G}}^{\mathrm{T}}+k_{\mathrm{ISC}}^{\mathrm{T}}}
$$

In the high-temperature limit, $k_{\text {ISC }}^{\mathrm{T}} \gg k_{\mathrm{P}}+k_{\mathrm{G}}^{\mathrm{T}}$. Hence, $\Phi_{\mathrm{S}}=\Phi_{\mathrm{S}}^{\infty}=\frac{A}{k_{\mathrm{P}}+k_{\mathrm{G}}^{\mathrm{T}}+A} \simeq 1$ (assuming that even in this range $A \gg k_{G}^{T}$ ), and EQUATION (14) becomes

$$
\left(\frac{\Phi_{\mathrm{DF}}}{\Phi_{\mathrm{PF}}}\right)_{\max }=\left(\frac{I_{\mathrm{DF}}}{I_{\mathrm{PF}}}\right)_{\max }=\frac{1}{\frac{1}{\Phi_{\mathrm{T}}}-1}
$$

For strong TADF to occur, the cycle $\mathrm{S}_{1} \rightarrow \mathrm{T}_{1} \rightarrow \mathrm{S}_{1}$ must repeat several times before photon emission or nonradiative decay can take place. To show this, it is convenient to present the TADF process as the following sequence (SCHEME 2):

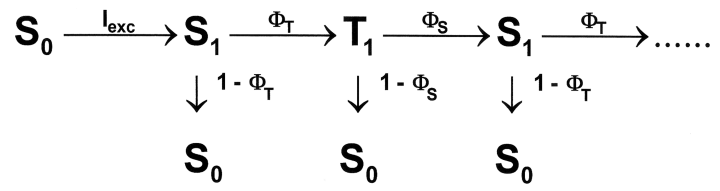

SCHEME 2. Sequential form for TADF. Taken from Ref. 26.

where the quantum yield of singlet formation is defined by EQUATION (15).

With this kinetic analysis, it is possible to determine several parameters. For example, the average number of cycles, $\bar{n}$, is given by

$$
\begin{aligned}
\bar{n} & =\sum_{n=0}^{\infty} n p_{\mathrm{n}}=\frac{\Phi_{\mathrm{T}} \Phi_{\mathrm{S}}}{1-\Phi_{\mathrm{T}} \Phi_{\mathrm{S}}} \\
= & \frac{1}{\frac{1}{\Phi_{\mathrm{T}} \Phi_{\mathrm{S}}}-1}=\frac{1}{\frac{1}{\Phi_{\mathrm{T}}}\left(1+\frac{1}{k_{\mathrm{ISC}}^{\mathrm{T}} \tau_{\mathrm{P}}^{0}}\right)-1}
\end{aligned}
$$

Comparison of EQUATIONS (17) and (14) gives immediately

$$
\frac{\Phi_{\mathrm{DF}}}{\Phi_{\mathrm{PF}}}=\frac{I_{\mathrm{DF}}}{I_{\mathrm{PF}}}=\bar{n}
$$

and, using EQUATION (13),

$$
\frac{\Phi_{\mathrm{F}}}{\Phi_{\mathrm{PF}}}=\frac{I_{\mathrm{F}}}{I_{\mathrm{PF}}}=1+\bar{n}
$$

hence, the increase in fluorescence intensity owing to TADF is a direct measure of the average number of $\mathrm{S}_{1} \rightarrow \mathrm{T}_{1} \rightarrow \mathrm{S}_{1}$ cycles performed. This result is easy to understand, because each return from $\mathrm{T}_{1}$ to $\mathrm{S}_{1}$ brings a new opportunity for fluorescence emission.

Without reversibility, $\bar{n}=0$. On the other hand, for the fastest possible excited state equilibration $\left(k_{\text {ISC }}^{\mathrm{T}}\right.$ $\rightarrow A, \Phi_{\mathrm{S}} \simeq 1$ ) one has

$$
\bar{n} \simeq \frac{1}{\frac{1}{\Phi_{\mathrm{T}}}-1}
$$

Therefore, the maximum possible fluorescence intensification factor, EQUATION (19), is $1 /\left(1-\Phi_{\mathrm{T}}\right)$.

Using the following set of data, obtained by our group for fullerene $\mathrm{C}_{70}$ dispersed in polystyrene $\mathrm{e}^{26,33,34}$ : $\Phi_{\mathrm{T}}=0.99, \tau_{\mathrm{F}}=630 \mathrm{ps}, \tau_{\mathrm{P}}^{0}=28 \mathrm{~ms}, A=8 \times 10^{7} \mathrm{~s}^{-1}$, $\Delta E_{\mathrm{ST}}=29 \mathrm{~kJ} \mathrm{~mol}^{-1}$, the maximum average number of cycles is estimated to be 99 , and the maximum fluorescence intensification factor to be 100 . The computed average number of cycles as a function of temperature is displayed in Figure 1. Many excited-state cycles are already effected at moderate temperatures.

Several methods of TADF data analysis exist. The classical one, from Parker, ${ }^{6}$ combines steady-state delayed fluorescence and phosphorescence intensities for the determination of $\Delta E_{\mathrm{ST}}$. This method was successfully applied to $\mathrm{C}_{70} .{ }^{8}$ Nevertheless, measuring the phosphorescence is often not possible or convenient, and it is precisely in these cases that a nonspectroscopic method for the estimation of $\Delta E_{\mathrm{ST}}$ becomes valuable. Furthermore, photophysical parameters other than $\Delta E_{\mathrm{ST}}$ are of interest and can be extracted from experimental TADF data by other methods.

From the steady-state data, and for curve fitting, we can conveniently rewrite EQUATION (14) as ${ }^{8}$ 


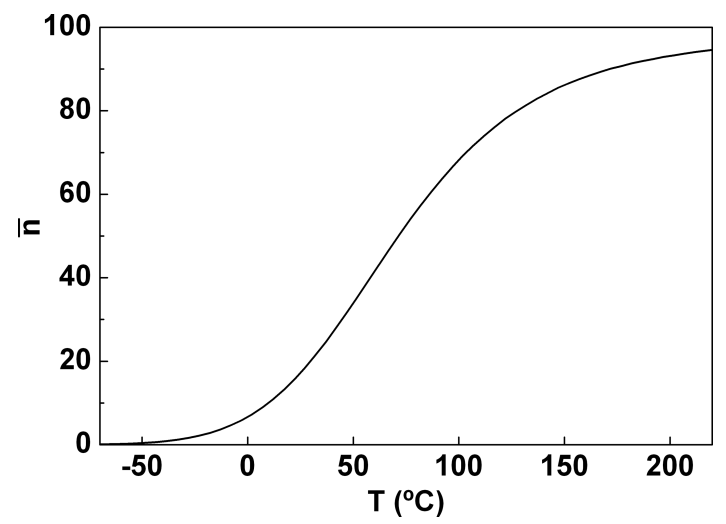

FIGURE 1. Computed average number of $\mathrm{S}_{1} \rightarrow \mathrm{T}_{1} \rightarrow$ $S_{1}$ cycles as a function of temperature for $C_{70}$ in polystyrene. Many excited-state cycles are already effected at moderate temperatures. From Ref. 26.

$$
\begin{aligned}
& \ln \left[\frac{I_{\mathrm{PF}}}{I_{\mathrm{DF}}}-\left(\frac{1}{\Phi_{\mathrm{T}}}-1\right)\right]= \\
& \ln \left[\frac{1}{\Phi_{\mathrm{T}}}\left(\frac{1}{\Phi_{\mathrm{S}}^{\infty}}-1\right)\right]+\frac{\Delta E_{\mathrm{ST}}}{R T}
\end{aligned}
$$

where

$$
\Phi_{\mathrm{S}}^{\infty}=\frac{1}{\frac{1}{A \tau_{\mathrm{P}}^{0}}+1}
$$

and from a fit to steady-state data arranged in the above form ${ }^{8}$ it is possible to recover $\Delta E_{\mathrm{ST}}, \Phi_{\mathrm{T}}$, and $\Phi_{\mathrm{S}}^{\infty}$, assuming that $\Phi_{\mathrm{S}}^{\infty}$ is temperature independent. Alternatively, a nonlinear curve fitting can also be carried out.

Concerning the time-resolved data, the time constant for the TADF lifetime is given by ${ }^{26}$

$$
\lambda_{1}=\frac{\frac{1}{\tau_{\mathrm{P}}^{0}}+k_{\mathrm{ISC}}^{\mathrm{T}}\left(1-\Phi_{\mathrm{T}}\right)}{1+k_{\mathrm{ISC}}^{\mathrm{T}} \tau_{\mathrm{F}}}
$$

and for $k_{\text {ISC }}^{\mathrm{T}} \tau_{\mathrm{F}} \ll 1$, as is usually the case, EQUATION (23) reduces to

$$
\lambda_{1}=\frac{1}{\tau_{\mathrm{P}}^{0}}+k_{\mathrm{ISC}}^{\mathrm{T}}\left(1-\Phi_{\mathrm{T}}\right)
$$

By using Equation (1), EQUATION (24) becomes

$$
\lambda_{1}=\frac{1}{\tau_{\mathrm{P}}^{0}}+B \exp \left(-\frac{\Delta E_{\mathrm{ST}}}{R T}\right)
$$

where $B=\left(1-\Phi_{\mathrm{T}}\right) A$. From a nonlinear fit to the temperature dependence of the fluorescence long component (delayed fluorescence lifetime) using EQUATION (25), and assuming that $\tau_{\mathrm{P}}^{0}$ is temperature independent, it is possible to recover $\Delta E_{\mathrm{ST}}, B$, and $\tau_{\mathrm{p}}^{0}$ from time-resolved measurements. Nevertheless, and owing to parameter correlation, it is preferable to fix $\Delta E_{\mathrm{ST}}$ at the steady-state value (obtained with EQUATION (21)). $A$ and $\tau_{\mathrm{P}}^{0}$ can thus be extracted from the temperature dependence of the delayed fluorescence lifetime. ${ }^{26} \mathrm{An}$ alternative procedure is to rewrite EQUATION (25) as

$$
\ln \left(\lambda_{1}-\frac{1}{\tau_{\mathrm{P}}^{0}}\right)=\ln B-\frac{\Delta E_{\mathrm{ST}}}{R T}
$$

and to search for the value of $\tau_{\mathrm{P}}^{0}$ that gives the best straight line.

A new method of analysis combines steady-state and time-resolved (delayed fluorescence) data in the same $\operatorname{plot}^{26}$ :

$$
\tau_{\mathrm{DF}}=\tau_{\mathrm{P}}^{0}-\left(\frac{1}{\Phi_{\mathrm{T}}}-1\right) \tau_{\mathrm{P}}^{0} \frac{I_{\mathrm{DF}}}{I_{\mathrm{PF}}}
$$

This linear plot yields $\Phi_{\mathrm{T}}$ and $\tau_{\mathrm{P}}^{0}$, assuming $\tau_{\mathrm{P}}^{0}$ to be temperature independent. If $\tau_{\mathrm{P}}^{0}$ is already known, $\Phi_{\mathrm{T}}$ can be directly obtained from EQUATION (27).

In conclusion, from steady-state and time-resolved data, it is in principle possible to obtain $\Phi_{\mathrm{T}}, A, \Delta E_{\mathrm{ST}}$, and $\tau_{\mathrm{p}}^{0}$ by using several methods.

\section{Sensing Applications}

The increasing need for continuous monitoring in areas as diverse as biotechnology, health care, environmental sciences, aerospace industry, nuclear industry, and marine sciences led to an increase of activity in the development of optical sensors beginning in the 1980s. Optical chemical sensors allow the continuous recording of the concentration of chemical species (e.g., $\mathrm{O}_{2}, \mathrm{CO}_{2}$, or several ions) and physical parameters (e.g., pressure, temperature) and therefore several applications have been found. Among the many optical methods that are used for sensing, fluorescence has attracted special attention because it is highly sensitive and versatile. ${ }^{35}$

In fluorescence, the sample can be both excited and measured optically. Therefore, fluorescence-based sensors, not requiring contact with the medium during measurement, are advantageous compared with contact sensors in applications where electromagnetic noise is strong or it is physically difficult to connect a wire. Further advantages of the molecular fluorescence sensors are the fast response, the reversibility, and the space resolution that can go from the macroscale (fluorescent paints) down to the nanoscale (fluorescence microscopy). These properties also overcome the limitations of electrochemical sensors (difficult to miniaturize, invasive technique, and limited to discrete points). 


\section{Temperature Measurement}

Temperature is a basic property of matter, and its measurement is often required for both scientific research and industrial applications. Real-time temperature monitoring is of paramount importance in industrial testing and manufacturing and in many biomedical diagnostic and treatment processes. There are several thermal sensors based on molecular optical properties, namely, luminescence. ${ }^{36}$ The use of fiber optics in conjugation with phosphors, whose luminescence lifetime changes with temperature, is a well-established method. ${ }^{37}$ More recently, several studies have been devoted to fluorescence molecular thermometry, ${ }^{38}$ including one molecular thermometer based on the fluorescence quenching of fullerene $\mathrm{C}_{60}$ dispersed in a polymer (polymethyl methacrylate) film. ${ }^{39}$

There is presently a need for optical sensors covering a wide temperature range, say, from $100^{\circ} \mathrm{C}$ to $250^{\circ} \mathrm{C}$ or even more. The common luminescence temperature sensors used currently are based on metallic complexes (e.g., $\mathrm{Ru}, \mathrm{Pt}, \mathrm{Pd}$ ) whose intensities almost invariably decrease with a temperature increase owing to thermally activated quenching processes, ${ }^{40}$ with working range temperatures below $100^{\circ} \mathrm{C}$. The high thermal stability and the unique photophysical properties of fullerenes make these molecules well placed to fulfill this need.

The discovery ${ }^{8}$ of the TADF in fullerene $\mathrm{C}_{70}$ was the first step for the development of temperature sensors based on the delayed fluorescence of fullerenes. The study was carried out in a degassed solution of liquid paraffin. The fluorescence spectra at different temperatures in degassed and nondegassed solutions are shown in Figure 2. ${ }^{8}$

The intensity of the nondegassed solution is independent of temperature and is entirely due to PF. The rise with temperature observed in the degassed solutions results from the increasing contribution of delayed fluorescence to the total intensity. The delayed fluorescence obtained at $70^{\circ} \mathrm{C}$ in degassed medium is 50 times stronger than the $\mathrm{PF}$.

Fister $e t a l .{ }^{41}$ demonstrated the use of the known delayed fluorescence of acridine yellow for the development of a molecular thermometer in the -50 to $+50^{\circ} \mathrm{C}$ range. However, in contrast to $\mathrm{C}_{70}$, the TADF of this compound is weak, which precludes fluorescence intensity measurements without the use of a time delay. Furthermore, the observed triplet decay is complex, preventing a clear interpretation of the results.

Other fullerenes molecules such as $\mathrm{C}_{60}{ }^{22}$ and some $\mathrm{C}_{60}$ derivatives, ${ }^{23,24}$ as well as one $\mathrm{C}_{70}$ derivative, ${ }^{25}$ also exhibit TADF, but not as strongly as $\mathrm{C}_{70}$ because of the high $\Delta E_{\mathrm{ST}}$ and the lower $\Phi_{\mathrm{T}}$ exhibited by these

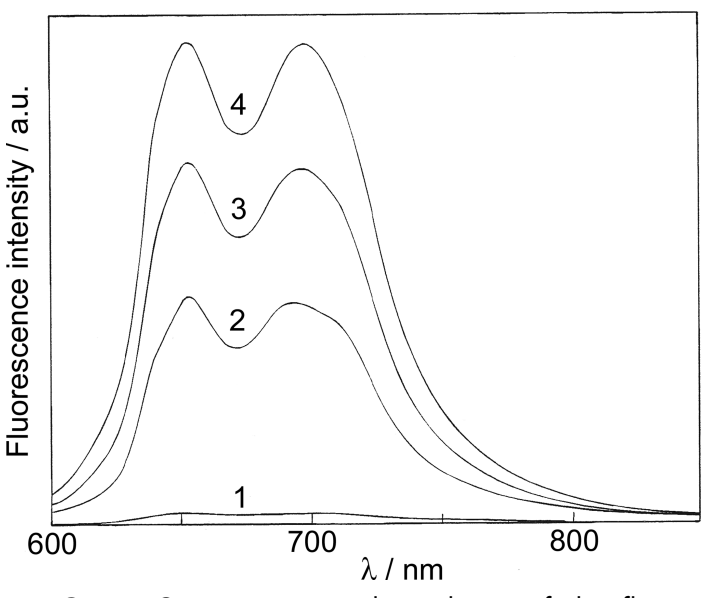

FIGURE 2. Temperature dependence of the fluorescence of a $\mathrm{C}_{70}$ solution in liquid paraffin. The emission of the aerated solution (1) is temperature independent, whereas a pronounced increase is observed upon degassing: $23^{\circ} \mathrm{C}$ $(2), 50^{\circ} \mathrm{C}(3)$, and $70^{\circ} \mathrm{C}(4)$. Adapted from Ref. 8.

molecules. For example, for fullerene $\mathrm{C}_{60}$, the global $\Phi_{\mathrm{F}}$ can in principle be, at most, 10 times higher than that of $\mathrm{PF}^{22}$ whereas for fullerene $\mathrm{C}_{70}$ that value can be 167 times. $^{8}$

The reversibility of the $\mathrm{C}_{70}$ /paraffin system was also evaluated. Up to $70^{\circ} \mathrm{C}$, the system shows total reversibility; however, for higher temperatures the reversibility is lost. Another drawback is the liquid nature of the system. For these reasons, we developed a series of polymer films with $\mathrm{C}_{70}$ molecularly dispersed in them. ${ }^{33,34}$

To study the influence of the polymer matrix structure on the photophysics and TADF of $\mathrm{G}_{70}$, we selected three polymers: polystyrene (PS), poly(tert-butyl methacrylate) (PtBMA), and poly(1-vinylnaphthalene) (PIVN). The films were prepared by evaporating a toluene solution of $\mathrm{C}_{70}$ and polymer on a quartz plate. After film formation and drying, the plates were placed in a quartz cell that was degassed at room temperature and afterward sealed. All the films exhibited absorption spectra similar to that of $\mathrm{C}_{70}$ in toluene (for PS and PIVN) or methylcyclohexane (for PtBMA). These results are in agreement with a molecular dispersion of $\mathrm{G}_{70}$ in the polymeric films.

The fluorescence of the $\mathrm{C}_{70} / \mathrm{PS}$ film at different temperatures and over a full heating-cooling cycle is shown in Figure 3. The first spectrum was recorded at room temperature $\left(25^{\circ} \mathrm{C}\right)$ before degassing and corresponds to PF. Without degassing, the fluorescence intensity is temperature independent. After degassing, a 22-fold enhancement of the room-temperature fluorescence was observed. This enhancement is a 


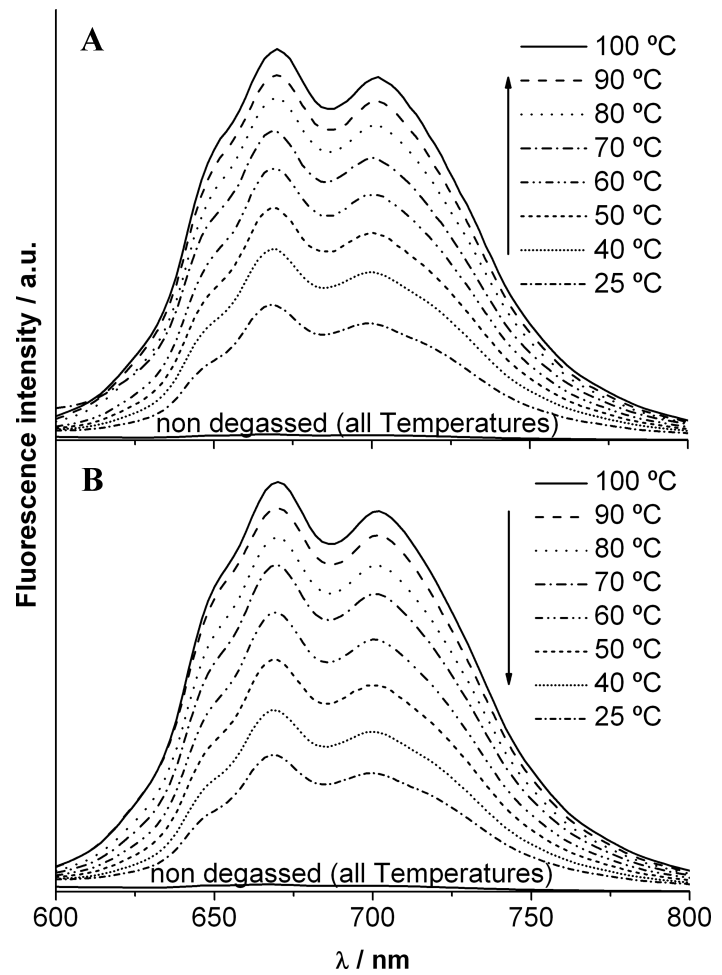

FIGURE 3. Fluorescence spectra $\left(\lambda_{\text {exc }}=470 \mathrm{~nm}\right)$ of a degassed $C_{70} / P S$ film at different temperatures: (A) heating sequence (from 25 to $100^{\circ} \mathrm{C}$ ); (B) cooling sequence (from 100 to $25^{\circ} \mathrm{C}$ ). The emission of the nondegassed sample is also shown for comparison. Taken from Ref. 34.

consequence of the additional contribution of delayed fluorescence to the overall emission. Heating the sample to $100^{\circ} \mathrm{C}$ (FIG. 3A) (a temperature at which the delayed fluorescence is 70 times higher than the $\mathrm{PF}$ ) shows that the fluorescence of $\mathrm{C}_{70}$ has a strong temperature dependence. The $\mathrm{C}_{70} / \mathrm{PS}$ film exhibits full reversibility and fluorescence intensity cycles without hysteresis. The results exhibited a high degree of reproducibility.

The values of $I_{\mathrm{DF}} / I_{\mathrm{PF}}$ for the $\mathrm{C}_{70} / \mathrm{PS}$ film at different temperatures are collected in TABLE 1 and compared with the values previously measured for a solution of $\mathrm{C}_{70}$ in liquid paraffin. ${ }^{8}$ The $I_{\mathrm{DF}} / I_{\mathrm{PF}}$ ratios for the $\mathrm{C}_{70}$ /PS film are always higher than the values reported for $\mathrm{C}_{70}$ in paraffin. The values of $I_{\mathrm{DF}} / I_{\mathrm{PF}}$ for the films were also measured over a wider temperature range than for $\mathrm{C}_{70}$ in paraffin. The stability of the $\mathrm{C}_{70} / \mathrm{PS}$ after long-term storage is also high, with comparable ratios ( $<2 \%$ variation) of $I_{\mathrm{DF}} / I_{\mathrm{PF}}$ being measured after several weeks of storage.

Identical temperature cycles were carried out for the $\mathrm{C}_{70} / \mathrm{P} 1 \mathrm{VN}$ and $\mathrm{C}_{70} / \mathrm{PtBMA}$ films. Responses similar
TABLE 1. Experimental $I_{\mathrm{DF}} / I_{\mathrm{PF}}(700 \mathrm{~nm})$ for the $C_{70}$ polymer systems for various temperatures

\begin{tabular}{lcccc}
\hline $\begin{array}{l}I_{\mathrm{DF}} / I_{\mathrm{PF}}(700 \mathrm{~nm}) \\
\text { system }\end{array}$ & $25^{\circ} \mathrm{C}$ & $50^{\circ} \mathrm{G}$ & $70^{\circ} \mathrm{C}$ & $100^{\circ} \mathrm{C}$ \\
\hline $\mathrm{C}_{70} /$ paraffin $^{a}$ & 20 & 35 & 50 & $-{ }^{b}$ \\
$\mathrm{C}_{70} / \mathrm{PS}$ & 22 & 39 & 53 & 70 \\
$\mathrm{C}_{70} / \mathrm{P} 1 \mathrm{VN}$ & 17 & 30 & 40 & 50 \\
$\mathrm{C}_{70} /$ PtBMA & 18 & 35 & 51 & 79 \\
\hline
\end{tabular}

${ }^{a}$ Ref. 34.

b_, no data.

to that of the $\mathrm{C}_{70} / \mathrm{PS}$ film were observed. The films exhibit good reversibility in the thermal cycles and high reproducibility. The $I_{\mathrm{DF}} / I_{\mathrm{PF}}$ values for these films at several temperatures are also reported in TABLE 1 . The maximum $I_{\mathrm{DF}} / I_{\mathrm{PF}}$ value was obtained at $100^{\circ} \mathrm{C}$ with the $\mathrm{C}_{70} /$ PtBMA system.

The temperature sensitivity of fluorescence intensity was also calculated and can be defined either as the variation of the fluorescence quantum yield with temperature, which is the absolute sensitivity $S_{A}$ (EQUATION (28)), or as the relative variation of the fluorescence quantum yield with temperature, which is the relative sensitivity $S_{R}$ (EQUATION (29)).

$$
\begin{gathered}
S_{A}=\frac{d \Phi_{F}}{d T} \\
S_{R}=\frac{1}{\Phi_{\mathrm{F}}} \frac{d \Phi_{\mathrm{F}}}{d T}=\frac{d \ln \Phi_{\mathrm{F}}}{d T}
\end{gathered}
$$

We will use the relative sensitivity because it directly reflects the relative variation of the fluorescence intensity. The temperature dependence of $S_{R}$ for the $\mathrm{C}_{70}$ /polymer systems is displayed in FIgURE 4 . The $\mathrm{C}_{70}$ /polymer systems have some of the highest temperature sensitivities known over a broad temperature range. ${ }^{42}$ To define a useful working range, we assume a minimum value of $0.5 \% \mathrm{~K}^{-1}$ for $S_{R}$. With this value, the lower temperature limit is $-80^{\circ} \mathrm{C}$ for all polymers. At the other end of the scale, the $\mathrm{C}_{70}$ /PtBMA system displays the highest high-temperature limit $\left(140^{\circ} \mathrm{C}\right)$, whereas for the other two polymers the upper limit is $110^{\circ} \mathrm{C}$.

The performance of $\mathrm{C}_{70} / \mathrm{PtBMA}$ was compared against ruthenium(II) polypyridyl complexes, which exhibit a strong temperature dependence. In particular, $\left[\mathrm{Ru}(\mathrm{phen})_{3}\right](\operatorname{tris}(1,10-$ phenantroline $) r u t h e n i u m)$ is a common optical temperature probe that displays efficient temperature quenching and high sensitivity. It is used because (1) it can be incorporated in solid matrices, such as sol-gels or polymers; (2) it is commercially available; (3) it is photostable; (4) it has a 


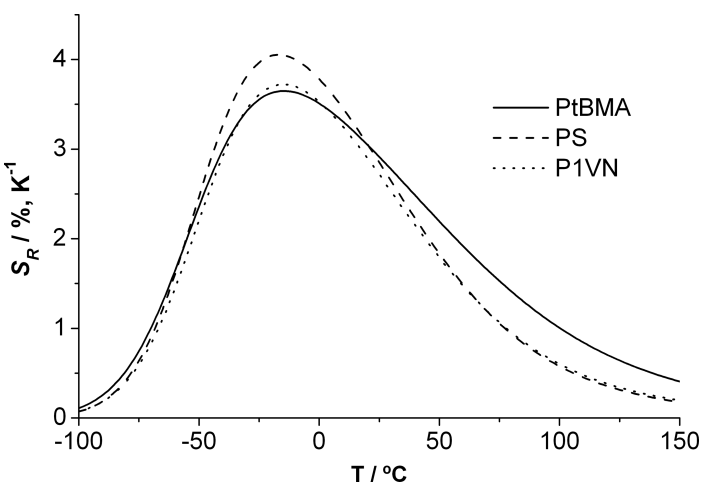

FIGURE 4. Relative variation in fluorescence $S_{R}$ versus temperature for $\mathrm{C}_{70} / \mathrm{PtBMA}$ (solid line), $\mathrm{C}_{70} / \mathrm{PS}$ (dashed line), and $C_{70} / P 1 V N$ (dotted line). Taken from Ref. 34.

large Stokes shift; and (5) it can be excited in the visible region. ${ }^{43}$ However, the luminescence of $\mathrm{Ru}(\mathrm{II})$ polypyridyl complexes is quenched by oxygen, and to avoid this interference in temperature sensing, we used poly(acrylonitrile) (PAN) as a matrix as a result of its very low gas permeability. ${ }^{44}$ The temperature dependence of the luminescence quantum yields of $\mathrm{C}_{70} / \mathrm{PtBMA}$ and $\left[\mathrm{Ru}(\mathrm{phen})_{3}\right] / \mathrm{PAN}$ systems are shown in Figure 5. For temperatures higher than $80^{\circ} \mathrm{C}$, the luminescence quantum yield of $\mathrm{C}_{70}$ in PtBMA exceeds that of $\left[\mathrm{Ru}(\mathrm{phen})_{3}\right]$ in PAN.

The $\mathrm{C}_{70}$-based luminescence thermometer is a new development in the molecular thermometry field owing to the possibility of using a highly sensitive probe that covers not only both the low temperature and the physiological temperature ranges but also temperatures well above $100^{\circ} \mathrm{C}$.

\section{Oxygen Sensing}

Oxygen, being essential for life, is an immensely important chemical species. Determination of oxygen levels is required in many different research areas. In medicine, oxygen levels in exhaled air or in the blood of a patient are key physiological parameters. Such parameters should ideally be monitored continuously. The measurement of oxygen levels is also essential in industries that use metabolizing organisms, such as yeast for brewing and baking, and in biotechnology, where microorganisms are used in the production of antibiotics and anticancer drugs.

Trace oxygen detection is important from a safety standpoint, ${ }^{45}$ because oxygen leaks can cause fires and explosions and can be harmful in storage chambers and packaged food ${ }^{46}$ and in aerospace research. ${ }^{47}$ Common trace oxygen sensors are based on amperometry (Clark electrodes). These are sensitive and applicable over a wide temperature range but are dif-

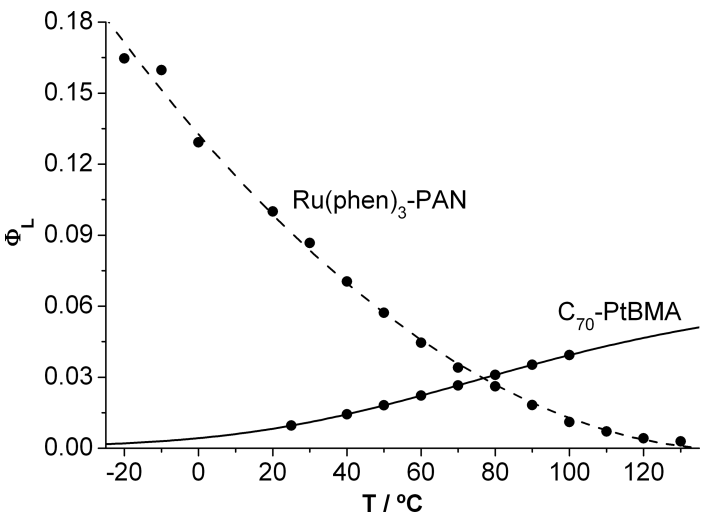

FIGURE 5. Temperature dependence of the luminescence quantum yields $\left(\Phi_{\mathrm{L}}\right)$ of $\mathrm{C}_{70} / \mathrm{PtBMA}$ (solid line) and $\left[\mathrm{Ru}\left(\right.\right.$ phen) $\left.{ }_{3}\right] /$ PAN (dashed line). Experimental points are shown as circles. Taken from Ref. 34.

ficult to miniaturize, invasive, and limited to discrete points. ${ }^{48}$

Optical sensors do overcome these limitations. Recently, a variety of devices and sensors based on molecular optical properties have been developed to measure oxygen partial pressure on the solid surface. Many optical oxygen sensors are composed of organic dyes, transition metal complexes, and metalloporphyrins immobilized in oxygen-permeable materials. ${ }^{49}$ There is still a need for optical sensors that can respond to low levels of oxygen. The sensitivity to oxygen of excited fullerenes was observed in the triplet-triplet quenching of fullerene polymeric films by using laser flash photolysis. ${ }^{50}$ The TADF effect in the fullerenes is sensitive to the presence of oxygen, ${ }^{8}$ leading to an efficient quenching of the intensity and lifetime of TADF. Because of this ultrasensitivity to oxygen, fullerenes are good candidates for sensing oxygen in low concentrations.

In a recent report, fullerene $\mathrm{G}_{70}$ was embedded in two highly permeable polymer membranes, an organosilica and an ethyl cellulose, and used as optical sensor for trace amounts of oxygen with detection limits in the part-per-billion volume (ppbv) range. ${ }^{51}$

The highest $\mathrm{O}_{2}$ permeabilities are displayed by silica-based polymers. But in all reports of sol-gels doped with unfunctionalized fullerenes, the fullerene was partially aggregated owing to formation of small clusters. ${ }^{52}$ These aggregates show largely reduced fluorescence intensities and lifetimes as a result of self-quenching. We have been able to incorporate $\mathrm{C}_{70}$ into an organically modified silica (OS) without significant aggregation by using a monomer where one alkoxy group is replaced by a phenyl ring. ${ }^{52}$ Organosilicas are less polar and thus more compatible with fullerenes. ${ }^{53,54}$ Ethyl cellulose $49 \%$ 
TABLE 2. Stern-Volmer constants and detection limits for delayed fluorescence quenching of $C_{70}$ in OS and EC, respectively, by $\mathrm{O}_{2}$ at various temperatures and comparison with the literature

\begin{tabular}{|c|c|c|c|c|}
\hline Probe & $\mathrm{T}\left({ }^{\circ} \mathrm{C}\right)$ & $\mathrm{K}_{\mathrm{SV} 1} /\left(\mathrm{mg}\left(\mathrm{O}_{2}\right) / \mathrm{L}\right)^{-1}$ & Detection limit/(ppmv $\left.\left(\mathrm{O}_{2}\right)\right)$ & Ref. \\
\hline \multirow[t]{4}{*}{$\mathrm{G}_{70} / \mathrm{OS}$} & 0 & 25.1 & 0.34 & 51 \\
\hline & 20 & 28.4 & 0.30 & 51 \\
\hline & 60 & 26.0 & 0.32 & 51 \\
\hline & 120 & 15.1 & 0.54 & 51 \\
\hline \multirow[t]{4}{*}{$\mathrm{G}_{70} / \mathrm{EG}$} & 0 & 47.0 & 0.18 & 51 \\
\hline & 20 & 52.9 & 0.16 & 51 \\
\hline & 60 & 36.1 & 0.23 & 51 \\
\hline & 120 & 16.9 & 0.48 & 51 \\
\hline $\mathrm{PdOEP} /$ silica & 20 & 3.74 & 2.44 & 58 \\
\hline $\mathrm{PtOEP} /$ silica & 20 & 0.37 & 25.26 & 58 \\
\hline PtTFPP/silica & 20 & 0.12 & 77.88 & 58 \\
\hline $\mathrm{PtTPP} /$ silica & 20 & 0.17 & 57.38 & 58 \\
\hline $\mathrm{Ru}(\mathrm{dpp})_{3} /$ silica & 20 & $3.2 \times 10^{-2}$ & 400.27 & 58 \\
\hline PdTFPP/PTBS & 20 & 1.32 & 6.87 & 55 \\
\hline PdTFPP/EC & 20 & 1.27 & 6.42 & 55 \\
\hline $\mathrm{PdTFPP} / \mathrm{EC}^{a}$ & 20 & 1.12 & 7.35 & 55 \\
\hline PtTFPP/PTBS & 20 & 0.13 & 77.31 & 55 \\
\hline PtTFPP/EG & 20 & 0.18 & 51.41 & 55 \\
\hline $\mathrm{Ru}(\mathrm{dpp})_{3} / \mathrm{PTBS}$ & 20 & $1.4 \times 10^{-2}$ & 776.33 & 55 \\
\hline
\end{tabular}

Taken from Ref. 51

${ }^{a}$ EC with 46\% ethylation; PdOEP: palladium octaethylporphyrin; PdTFPP: palladium tetrapentafluorophenylporphyrin; PtOEP: platinum ocatethylporphyrin; PTBS: poly (4-tert)butyl styrene; PtTFPP: platinum tetrapentafluorophenylporphyrin; PtTPP: platinum tetraphenylporphyrin; $\mathrm{Ru}(\mathrm{dpp}) 3$ : ruthenium tris-(4,7)-diphenylphenanthroline.

(EC) also is a highly permeable matrix for oxygen sensing. ${ }^{55} \mathrm{C}_{70}$ is compatible with this matrix. The absence of significant aggregation was demonstrated through the absorption spectra, lifetime measurements, atomic force microscopy, and scanning electron microscopy. ${ }^{51}$

We investigated the sensitivity to oxygen by time domain fluorescence lifetime imaging. ${ }^{56}$ The delayed fluorescence lifetimes exceed $20 \mathrm{~ms}$ in the absence of oxygen at room temperature and below and result in an extreme sensitivity to oxygen. The response is instantaneous $(<0.1 \mathrm{~s})$. The best fits for the Stern-Volmer plots were obtained by applying the two-site quenching model. ${ }^{57}$ The fluorescence is most pronounced at $120^{\circ} \mathrm{C}$, and $\mathrm{C}_{70}$ still shows delayed fluorescence lifetimes greater than $5 \mathrm{~ms}$. The temperature dependence of the sensitivity is therefore the result of the following three effects upon increasing temperature: (1) increasing $\Phi_{\mathrm{DF}}$, (2) decreasing delayed fluorescence lifetime, and (3) higher collision rate of $\mathrm{O}_{2}$. The Stern-Volmer constants depend on temperature in a nonlinear way, and both systems display detection limits (defined at $1 \%$ quenching) more than one order of magnitude better than state-of-the-art probes (TABLE 2).

The response of the matrices is fully reversible over many hundreds of times and showed no detectable degradation after 3 months of storage at room temper- ature in the dark on air. In conclusion, we developed an optical oxygen sensor that is especially suited for sensing oxygen down to the ppbv range and at high temperatures. The method makes use of the TADF of fullerene $\mathrm{C}_{70}$ dissolved in appropriate polymers. It enables, for the first time, the optical sensing and imaging of oxygen at the ppbv level, and thus has a large potential.

\section{Concluding Remarks and Future Perspectives}

This report reviewed selected results of our work in fullerene photophysics, with an emphasis on TADF. We showed that fullerenes display a strong TADF effect, which can be used to determine several photophysical parameters. This effect also allows the use of fullerenes as temperature and oxygen optical sensors under extreme conditions (high temperatures $\left[>100^{\circ} \mathrm{C}\right]$ or low oxygen concentration $[<1$ part-per-million volume (ppmv)]).

Despite the work already carried out, knowledge of the photophysics of fullerenes and derivatives is still incomplete, and much remains to be done in this area and in optical sensor systems incorporating fullerenes.

The development of new materials with ultralow (or even zero) oxygen permeability and high thermal 
stability (several hundreds of degrees) are needed to improve the optical temperature sensor materials based on fullerenes and TADF. These materials will bypass the drawback of working under vacuum, which is mandatory for using TADF as the mechanism of detection.

For optical sensors, another issue that should be given attention is the sensing of rare gases. The photophysics of fullerenes is affected by the external heavyatom effect, such as that of bromine and iodine, ${ }^{59,60}$ and it is expected that rare gases like krypton and xenon will have similar quenching properties.

The synthesis and preparation of new host-guest supramolecular assemblies, incorporating fullerenes and gold nanoparticles, has been subject of interest in the last few years. ${ }^{61}$ These materials are especially suited for light-energy conversion systems. However, metal nanoparticles (e.g., those made of gold and silver) can tune the luminescence of fluorophores. ${ }^{62,63}$ These variations are highly dependent on the diameter of the nanoparticles or type of surface $e^{64}$ and can in principle be used in more complex sensor systems for multiparameter analysis.

Efforts should be made to obtain new compounds and materials based on fullerenes and nanoparticles to measure relevant physical parameters and analytes under favorable conditions.

\section{Acknowledgments}

This work was supported by Fundação para a Ciência e a Tecnologia (FCT, Portugal) and POCI 2010 (FEDER) within project POCI/QUI/58535/2004. C. Baleizão was supported through fellowship SFRH/BPD/28438/2006. Collaboration with the group of O. S. Wolfbeis (Institute of Analytical Chemistry, Chemo-, and Biosensors, University of Regensburg, Germany) is gratefully acknowledged.

\section{Conflict of Interest}

The authors declare no conflicts of interest.

\section{References}

1. Kroto, H.W. et al. 1985. $\mathrm{C}_{60}$-buckminsterfullerene. Nature 318: $162-163$.

2. KRÄtschmer, W. et al. 1990. Solid $\mathrm{C}_{60}$-a new form of carbon. Nature 347: 354-358.

3. Foотe, G.S. 1994. Photophysical and photochemical properties of fullerenes. Top. Curr. Chem. 169: 347-363.
4. Sun, Y.P. 1997. Photophysics and photochemistry of fullerene materials. In Molecular and Supramolecular Photochemistry, Vol. 1, Organic Photochemistry. V. Ramamurthy \& K.S. Shanze, Eds.: 325-390. Marcel Dekker. New York.

5. Sun, Y.P. et al. 2000. Photoexcited state and electron transfer properties of fullerenes and related materials. In Optical and Electronic Properties of Fullerenes and FullereneBased Materials. J. Shinar, Z.V. Vardeny \& Z.H. Kafafi, Eds.: 43-81. Marcel Dekker. New York.

6. Parker, C.A. 1968. Photoluminescence of Solutions. Elsevier. Amsterdam.

7. Valeur, B. 2002. Molecular Fluorescence: Principles and Applications. Wiley-VCH. Weinheim.

8. Berberan-Santos, M.N. \& J.M.M. Garcia. 1996. Unusually strong delayed fluorescence of $\mathrm{C}_{70} . \mathrm{J}$. Am. Chem. Soc. 118: $9391-9394$.

9. LAM, S.K. \& D. Lo. 1997. Time-resolved spectroscopic study of phosphorescence and delayed fluorescence of dyes in silica-gel glasses. Chem. Phys. Lett. 281: 35-43.

10. Duchowicz, R., M.L. Ferrer \& A.U. Acuña. 1998. Kinetic spectroscopy of erythrosin phosphorescence and delayed fluorescence in aqueous solution at room temperature. Photochem. Photobiol. 68: 494-501.

11. Wolf, M.W. et al. 1975. Photophysical studies on the benzophenones. Prompt and delayed fluorescences and selfquenching. J. Am. Chem. Soc. 97: 4490-4497.

12. Turek, A.M. et al. 2002. Resolution of benzophenone delayed fluorescence and phosphorescence with compensation for thermal broadening. J. Phys. Chem. A 106: 6044 6052 .

13. Maciejewski, A., M. Szymanski \& R.P. Steer. 1986. Thermally activated delayed S1 fluorescence of aromatic thiones. J. Phys. Chem. 90: 6314-6318.

14. Eisenberger, H. \& B. Nickel. 1996. Photophysical triplet state processes of 4-H-1-benzopyrane-4-thione in a perfluoroalkane. Part 1.- Temperature dependence of unimolecular triplet decay. J. Chem. Soc. Faraday Trans. 92: 733-740.

15. Yusa, S., M. Kamachi \& Y. Morishima. 1998. Photophysical behavior of zinc(II) tetraphenylporphyrin covalently incorporated in a cholesterol-bearing polymethacrylate. Photochem. Photobiol. 67: 519-525.

16. KRopp, J.L. \& W.R. DAwson. 1967. Radiationless deactivation of triplet coronene in plastics. J. Phys. Chem. 71: 4499-4506.

17. Nickel, B. \& D. Klemp. 1993. The lowest triplet state of azulene- $h_{8}$ and azulene- $d_{8}$ in liquid solution. I. Survey, kinetic considerations, experimental technique, and temperature dependence of triplet decay. Chem. Phys. 174: 297-318.

18. Nickel, B. \& D. KLemp. 1993. The lowest triplet state of azulene- $h_{8}$ and azulene- $d_{8}$ in liquid solution: II. Phosphorescence and E-type delayed fluorescence. Chem. Phys. 174: $319-330$.

19. Arbogast, J.W. \& C.S. Foote. 1991. Photophysical properties of $\mathrm{C}_{70}$. J. Am. Chem. Soc. 113: 8886-8889.

20. Argentine, S.M., K.T. Kotz \& A.H. Francis. 1995. Temperature and solvent effects on the luminescence spectrum of $\mathrm{C}_{70}$ : assignment of the lowest singlet and triplet states. J. Am. Chem. Soc. 117: 11762-11767. 
21. WASIELEWSKI, M.R. et al. 1991. Triplet states of fullerenes $\mathrm{C}_{60}$ and $\mathrm{C}_{70}$. Electron paramagnetic resonance spectra, photophysics, and electronic structures. J. Am. Chem. Soc. 113: $2774-2776$.

22. Salazar, F.A., A. Fedorov \& M.N. Berberan-Santos, 1997. A study of thermally activated delayed fluorescence in $\mathrm{C}_{60}$. Chem. Phys. Lett. 271: 361-366.

23. Gigante, B. et al. 1999. Diels-Alder adducts of $\mathrm{C}_{60}$ and resin acid derivatives: synthesis, electrochemical and fluorescence properties. Tetrahedron 55: 6175-6182.

24. Anthony, S.M., S.M. Bachilo \& R.B. Weisman. 2003. Comparative photophysics of $\mathrm{C}_{61} \mathrm{H}_{2}$ isomers. J. Phys. Chem. A 104: 10674-10679.

25. BACHILO, S.M. et al. 2000. Time-resolved thermally activated delayed fluorescence in $\mathrm{C}_{70}$ and 1,2- $\mathrm{C}_{70} \mathrm{H}_{2}$. J. Phys. Chem. A 104: 11265-11269.

26. Baleizão, C. \& M.N. Berberan-Santos. 2007. Thermally activated delayed fluorescence as a cycling process between excited singlet and triplet states: application to the fullerenes. J. Chem. Phys. 126: 204510.

27. Tanaka, F., M. Okamoto \& S. Hirayama. 1995. Pressure and temperature dependences of the rate constant for S1$\mathrm{T} 2$ intersystem crossing of anthracene compounds in solution. J. Phys. Chem. 99: 525-530.

28. Berberan-Santos, M.N. \& J.M.G. Martinho. 1992. A linear response approach to kinetics with time-dependent rate coefficients. Chem. Phys. 164: 259-269.

29. Graves, W.E., R.H. HofeldT \& S.P. MaGlynN 1972. Temperature dependence of phosphorescence characteristics of aromatic hydrocarbons in poly(methylmethacrylate). J. Chem. Phys. 56: 1309-1314.

30. Berberan-Santos, M.N. \& J.M.G. Martinho. 1991. Diffusion-influenced excimer formation kinetics. J. Chem. Phys. 95: 1817-1824.

31. Birks, J.B. 1970. Photophysics of Aromatic Molecules. Wiley. London.

32. Rae, M. \& M.N. Berberan-SAntos. 2002. Pre-equilibrium approximation in chemical and photophysical kinetics. Chem. Phys. 280: 283-293.

33. Baleizão, C. \& M.N. Berberan-Santos. 2006. A molecular thermometer based on the delayed fluorescence of $\mathrm{C}_{70}$ dispersed in a polystyrene film. J. Fluoresc. 16: 215-219.

34. Baleizão, C. et al. 2007. Optical thermometer based on the delayed fluorescence of $\mathrm{C}_{70}$. Chem. Eur.J. 13: 3643-3651.

35. WolfBEIS, O.S. 2004. Optical technology until the year 2000: an historical overview. In Optical Sensors for Industrial, Environmental and Clinical Applications. R. Narayanaswamy \& O.S. Wolfbeis, Eds.: 1-34. Springer. Berlin.

36. Uchiyama, S., A.P. DE Silva \& K. Iwau. 2006. Luminescent molecular thermometers. J. Chem. Educ. 83: 720-727.

37. Grattan, K.T. \& Z.Y. Zhang. 1995. Fiber Optic Fluorescence Thermometry. Chapman and Hall. London.

38. Lou, J.F. et al. 1999. Fluorescence-based thermometry: Principles and applications. Rev. Anal. Chem. 18: 235-284.

39. AmaO, Y. \& I. OkURA. 2002. Optical molecular thermometer based on the fluorescence of fullerene dispersed in poly(methyl methacrylate) film. Bull. Chem. Soc. Jpn. 75: 389-391.
40. Demas, J.N. \& B.A. DeGrafF. 2001. Applications of luminescent transition platinum group metal complexesto sensor technology and molecular probes. Coord. Chem Rev. 211: 317-351.

41. Fister III, J.C., D. Rank \& J.M. Harris. 1995. Delayed fluorescence optical thermometry. Anal. Chem. 67: 4269 4275.

42. WolfBeis, O.S. 2004. Fiber-optic chemical sensors and biosensors. Anal. Chem. 76: 3269-3284.

43. Liebsch, G., I. Klimant \& O.S. Wolfbeis. 1999. Luminescence lifetime temperature sensing based on sol-gels and poly(acrylonitrile)s dyed with ruthenium metal-ligand complexes. Adv. Mater. 11: 1296-1299.

44. Brandrup, J., E.H. Immergut \& E.A. Grulke. 1999. Permeability and diffusion data. In Polymer Handbook. Wiley. New York.

45. Denison, D.M. et al. 1968. Problem of fire in oxygen-rich surroundings. Nature 218: 1110-1113

46. Mills, A. 2005. Oxygen indicators and intelligent inks for packaging food. Chem. Soc. Rev. 34: 1003-1011.

47. SEIFE, C. 2003. NASA's hypersonic lab studies factors leading to breakup. Science 299: 1971.

48. Ramamoorthy, R., P.K. Dutta \& S.A. Akbar. 2003. Oxygen sensors: materials, methods, designs and applications. J. Mater. Sci. 38: $4271-4282$.

49. Amao, Y. 2003. Probes and polymers for optical sensing of oxygen. Mikrochim. Acta 143: 1-12.

50. AmaO, Y., K. Asai \& I. OkuRA. 1999. Optical oxygen pressure sensing based on triplet-triplet quenching of fullerene-polystyrene film using laser flash photolysis: soccerballene $\mathrm{C}_{60}$ versus rugbyballene $\mathrm{C}_{70}$. Bull. Chem. Soc. Jpn. 72: 2223-2227.

51. NAGL, S. et al. 2007. Optical sensing and imaging of trace oxygen with record response. Angew. Chem. Int. Ed. Engl. 46: 2317-2319.

52. Brusatin, G. \& P. Innocenzi. 2001. Fullerenes in sol-gel materials. J. Sol-Gel Sci. Technol. 22: 189-204.

53. Klimant, I. et al. 1999. Fast response oxygen micro-optodes based on novel soluble Ormosil glasses. Mikrochim. Acta 131: $35-46$.

54. JARONIEC, M. 2006. Materials science: organosilica the conciliator. Nature 442: 638-640.

55. Apostolidis, A. et al. 2004. A combinatorial approach for development of materials for optical sensing of gases. J. Comb. Chem. 6: 325-331.

56. Lin, Z. et al. 2004. Fluorescent imaging of citrate and other intermediates in the citric acid cycle. Angew. Chem. Int. Ed. Engl. 43: 1735-1738.

57. Demas, J.N. \& B.A. DeGraff. 1995. Modeling of luminescence quenching-based sensors: comparison of multisite and nonlinear gas solubility models. Anal. Chem. 67: 1377-1380.

58. Han, B.H., I. Manners \& M.A. WinniK. 2005. Oxygen sensors based on mesoporous silica particles on layer-bylayer self-assembled films. Chem. Mater. 17: 3160-3171.

59. RAE, M. et al. 2006. Intra- and intermolecular heavy-atom effects on the fluorescence properties of brominated $\mathrm{C}_{60}$ polyads. J. Phys. Chem. B 110: 12809-12814.

60. Foley, S. et al. 2001. Effect of halogenated compounds on the photophysics of $\mathrm{C}_{70}$ and a monoadduct of $\mathrm{C}_{70}$ : some 
implications on optical limiting behaviour. Chem. Phys. 263: $437-447$.

61. IMAHORI, H. et al. 2005. Host-guest interactions in the supramolecular incorporation of fullerenes into tailored holes on porphyrin-modified gold nanoparticles in molecular photovoltaics. Chem. Eur. J. 11: 7265-7275.

62. Aslan, K. et al. 2007. Fluorescent core-shell Ag@ $\mathrm{SiO}_{2}$ nanocomposites for metal-enhanced fluorescence and sin- gle nanoparticle sensing platforms. J. Am. Chem. Soc. 16: 55-62.

63. Aslan, K., S.N. Malyn \& C.D. Geddes 2007. Metalenhanced fluorescence from gold surfaces: angular dependent emission. J. Fluoresc. 17: 7-13.

64. AsLAN, K. et al. 2005. Metal-enhanced fluorescence: an emerging tool in biotechnology. Curr. Opin. Biotech. 16: 55-62. 